\title{
Minimal Hepatic Encephalopathy (MHE)
}

\author{
Daniela Benedeto-Stojanov and Dragan Stojanov \\ Faculty of Medicine, University of Nis
}

Serbia

\section{Introduction}

Hepatic encephalopathy (HE) reflects a spectrum of neuropsychiatric abnormalities seen in patients with liver dysfunction after exclusion of other known brain disease.

Classification of hepatic encephalopathy is based on the clinical setting in which symptoms occur (Table 1). ${ }^{1}$ The encephalopathy of acute liver failure shares clinical characteristics with that of cirrhosis, but also exhibits unique features. In cirrhosis, three major syndromes can be present:

1. Episodic (precipitant-induced) encephalopathy, commonly seen in the hospital setting, where a superimposed event is a key factor.

2. Persistent (chronic) encephalopathy, seen with extensive portal-systemic shunts and after portocaval shunt surgery or placement of transjugular intrahepatic portosystemic shunt (TIPS).

3. Minimal (subclinical) encephalopathy reflects alterations in cognitive function in patients who clinically exhibit a normal mental state.

\begin{tabular}{llll}
\hline & $\begin{array}{l}\text { Hepatic } \\
\text { failure }\end{array}$ & $\begin{array}{l}\text { Extrahepatic } \\
\text { portal-systemic } \\
\text { shunting }\end{array}$ & Special features \\
\hline Acute liver failure & Maximal & Absent & $\begin{array}{l}\text { Development of brain oedema and } \\
\text { intracranial hypertension }\end{array}$ \\
Cirrhosis & Variable & Variable & $\begin{array}{l}\text { Low-grade cerebral oedema without } \\
\text { overt signs of intracranial hypertension } \\
\text { Precipitant induced }\end{array}$ \\
$\begin{array}{l}\text { Episodic } \\
\text { encephalopathy }\end{array}$ & Variable & Genarally large & $\begin{array}{l}\text { Most often seen after portocaval } \\
\text { surgery or TIPS } \\
\text { encephalopathy }\end{array}$ \\
$\begin{array}{l}\text { Minimal } \\
\text { encephalopathy }\end{array}$ & Variable & Variable & $\begin{array}{l}\text { Requires neuropsychological/ } \\
\text { neurophysiological testing }\end{array}$ \\
\hline
\end{tabular}

TIPS, transjugular intrahepatic portosystemic shunt.

Table 1. Classification of hepatic encephalopathy. 
Minimal hepatic encephalopathy (MHE), the mildest form of HE, is characterized by subtle motor and cognitive deficits, and impairs health-related quality of life (HRQOL). ${ }^{2}$

Cirrhotic patients with MHE have a normal neurological and mental status by the standards of clinical examination, yet demonstrate quantifiable neuropsychological defects. ${ }^{3}$ The term MHE refers to the subtle changes in cognitive function, electrophysiological parameters, cerebral neurochemical/neurotransmitter homeostasis, cerebral blood flow, metabolism, and fluid homeostasis that can be observed in patients with cirrhosis who have no clinical evidence of hepatic encephalopathy. 4

MHE has been described previously using several different names, such as, early, lowgrade, latent or subclinical HE to identify patients with subtle cognitive function abnormalities.

These subtle neurocognitive abnormalities primarily affect attention, speed of information processing, and motor abilities and coordination that are not recognizable on standard neurological examination. These neurocognitive abnormalities are independent of sleep dysfunction or problems with overall intelligence. . $^{5}$

It has been well-described that MHE has a subtle but negative impact on a patient's spatial skills, motor skills, the ability to perform complex tasks such as driving, and even quality of life. ${ }^{3-5}$ MHE predicts the development of overt HE and is associated with poor survival ${ }^{2}$. Its negative impact on daily living, among other reasons, has led some authors to suggest that the failure to diagnose this condition could be classified as a medical error., 910

\section{Epidemiology}

There are no accurate data on the incidence of HE. HE is classified as: overt hepatic encephalopathy (OHE) and MHE.

OHE occurs in $30 \%$ to $45 \%$ of cirrhotic patients ${ }^{11}$ and in $10 \%$ to $50 \%$ of patients with TIPS 12 and can be clinically diagnosed.

The true prevalence of MHE in patients with portal hypertension is inknown. MHE has been diagnosed in patients with liver cirrhosis and in patients with noncirrhotic portal hypertension. The prevalence of MHE has been reported in as many as $20 \%-84 \%$ of cirrhotics, depending on which methods or tools are used and fixed diagnostic cut-offs. ${ }^{2,3}$ Large variations in the prevalence of MHE are related to prior episode of OHE, severity of liver disease, age, preasence of esophageal varices, and surgical porto-systemic shunts. Patients who develop MHE are older, more often have alcohol as etiology of cirrhosis, have history of overt HE in the past, have more severe liver disease, and more often have esophagogastric varices. ${ }^{2,13}$

\section{Pathogenesis}

Despite much scientific research, the exact pathophysiological mechanisms leading to HE are not clearly understood. The most widely accepted theory of the pathogenesis of HE is that nitrogenous substances derived from the gut adversely affect the cerebral function. 


\subsection{Ammonia}

There are various explanations why liver dysfunction or portosystemic shunting might lead to encephalopathy. In healthy subjects, intestinal neurotoxins, such as ammonia, manganase and the benzodiazepine-GABA system generated by gut bacteria from food, are transported by the portal vein to the liver, where $80-90 \%$ is metabolized and/or excreted immediately. In all subtypes of hepatic encephalopathy this process is impaired, either because the hepatocytes are incapable of metabolizing the neurotoxins or because portal venous blood bypasses the liver through collateral circulation or a medically constructed shunt. Neurotoxins accumulate in the systemic circulation. Ammonia plays a key role in the pathogenesis of HE. The small molecules of ammonia cross the blood-brain barrier and are absorbed and metabolized by astrocytes, population of cells in the brain that constitutes $30 \%$ of the cerebral cortex. Alzheimer type II astrocytes are the only cells containing glutamine synthetase that metabolize ammonia. It is hypothesed that glutamine synthesis within the astrocytes causes brain swelling. ${ }^{14,15}$ Astrocytes also provide physical and nutritional support for neurons, maintain the integrity of the blood-brain barrier and regulate cerebral blood flow. ${ }^{16}$ Ammonia also modulates glutamate neurotransmission and induces neurosteroid production in neurons, leading to a positive modulatory effect on the gammaaminobutyric acid-A receptor. ${ }^{17}$ The precise molecular mechanism(s) responsible for neurological alteration in HE are not known. HE is associated with alterations in the expression of astrocytic and neuronal genes that code for various proteins that play a critical role in central nervous system function including maintenance of cell volume and neurotransmission. ${ }^{14}$

The pathogenesis of MHE is similar to that of HE. ${ }^{18-22}$ An increase in brain glutamine and brain water is pathophysiological change associated with deterioration in neuropsychological performance. Alterations in cerebral blood flow and glucose metabolism induced by ammonia are associated with a significant decrease of glucose utilization by various cortical regions that are involved in cognitive functions. ${ }^{21}$ The cerebral metabolic rate for ammonia and the permeability-surface area product for ammonia are significantly higher in patients with MHE. ${ }^{21}$ The increased permeability-surface area product of the blood-brain barrier permits ammonia to diffuse across the blood-brain barrier into the brain more freely than normal. This may cause ammonia-induced encephalopathy even though arterial ammonia levels are normal or near normal.

Cognitive deficits observed in patients with noncirrhotic portal hypertension have also been linked to ammonia. ${ }^{18}$ Patients with noncirrhotic portal hypertension, such as extrahepatic portal venous obstruction, exhibited abnormalities in the results of neuropsychological tests, oral glutamine challenge test, and magnetic resonance (MR) imaging and spectroscopy similar to those described in $\mathrm{HE}$ associated with cirrhosis. ${ }^{22}$

Other waste products implicated in hepatic encephalopathy include mercaptans (substances containing a thiol group), short-chain fatty acids and phenol.23

\subsection{Serotonin}

Serotonin, a neurotransmitter with widespread distribution in the CNS, is important for the regulation of sleep, circadian rhythmicity and locomotion. Changes in the synthesis, metabolism, storage and release of neuronal serotonin in HE suggest a serotonergic synaptic 
deficit. Serotonin metabolism is exquisitely and selectively sensitive to the degree of portosystemic shunting and hyperammonaemia, suggesting a role for serotonin in early neuropsychiatric symptoms of HE. ${ }^{24}$

\subsection{Branched-chain amino acids (BCAA) and false neurotransmitters}

An imbalans between aromatic aminoacids (AAA) (phenylalanine, tryptophan and tyrosine) and branched-chain amino acids (BCAA)(leucine, isoleucine and valine) has been described in patients with severe liver dysfunction. AAA and BCAA share a common transport mechanism into the CNS. AS a consequence of increased concentration of AAA, neuronal levels may be raised leading to the production of false neurotransmitters (octopamide and phenylethanolamide) ${ }^{25}$ with subsequent development of HE. ${ }^{26}$

\subsection{Zink}

Zinc is a substrate of urea cycle enzymes. It may be depleted in patients with cirrhosis. Zinc supplementation increases the activity of ornithine transcarbamalyse increasing excretion of ammonia ions. There is conflicting clinical data regarding zinc supplementation in the management of HE. $27,28,29$

\subsection{Manganese}

Manganese is a neurotoxin that accumulates in the brains of patients with cirrhosis and portosystemic shunts. ${ }^{30,31}$ Levels of manganase correlate with hyperintensity of nucleus pallidus seen on MR brain scans of patients with cirrhosis. These patients may also demonstrate extrapyramidal signs, suggesting that altered homeostasis of manganese and other minerals could be responsible for the cognitive deficits associated with liver cirrhosis.

\subsection{Systemic inflammatory response}

Iinflammatory response may be important factor that may contribute to the development of MHE and its progression to OHE. Inflammation elsewhere in the body may precipitate encephalopathy through the action of cytokines and bacterial lipopolysaccharide on astrocytes. ${ }^{32}$ A recent study reported that severity of MHE was independent of severity of liver disease and levels of blood ammonia but markers of inflammation (higher neutrophil counts, C-reactive protein levels, and interleukin-6 levels) were significantly higher in those with MHE compared to those without MHE.33 Same authors showed that induced hyperammonemia resulted in significantly greater deterioration in psychometric tests in cirrhotic patients who had an ongoing infection compared with those in whom the infection had resolved. ${ }^{34}$ These two studies suggest that inflammation plays a synergistic role with ammonia in producing and modulating MHE.

\subsection{Intestinal flora}

Intestinal flora and endotoxins are another link between inflamation, ammonia and MHE. Imbalance of intestinal flora among cirrhotics compared to normal healthy controls has been demonstrated in the study of Zhao et al. ${ }^{35}$ They found increase in the counts of aerobes (such as Enterobacter and Enterococcus) and anaerobes (such as Clostridium) and a decrease in 
the count of Bifidobacterium. The severity of imbalance in intestinal flora matched the degree of liver dysfunction. Liu et al. ${ }^{36}$ reported that cirrhotic patients with MHE had substantial derangements in the gut microecology, with significant fecal overgrowth of potentially pathogenic Escherichia coli and Staphylococcus species. Treatment with synbiotics significantly increased the fecal content of non-urease-producing Lactobacillus species at the expense of these other bacterial species. Such modulation of gut flora was associated with a significant reduction in blood ammonia levels and reversal of MHE in 50\% of patients. Synbiotic treatment was also associated with a significant reduction in endotoxemia. The CTP functional class improved in nearly $50 \%$ of the patients.

\section{Clinical characteristics}

OHE is traditionally classified into four grades according to the West Haven criteria (Table 2). ${ }^{6}$

\begin{tabular}{ll}
\hline Grade 0 & Lack of detectable changes in personality or behavior \\
Grade 1 & No asterixis \\
& Trivial lack of awareness \\
& Euphoria or anxiety \\
& Shortened attention span \\
& Impaired performance of addition \\
& Asterixis may be present \\
& Lethargy or apathy \\
Grade 2 & Minimal disorientation for time or place \\
& Subtle personality change \\
& Inappropriate behavior, slurred speech \\
& Impaired performance of subtraction \\
& Asterixis is present \\
& Somnolence to semi-stupor, but responsive to verbal stimuli \\
Grade 3 & Confusion \\
& Gross disorientation \\
Grade 4 & Asterixis is usually absent \\
& Coma (unresponsive to verbal or noxious stimuli) \\
\hline
\end{tabular}

Adapted from Mullen KD6

Table 2. West Haven criteria for semiquantitative grading of mental state

Patients with MHE have a normal neurological examination; however they may still be symptomatic. Symptoms relate to disturbances in sleep, memory, attention, concentration and other areas of cognition. ${ }^{37,38}$ A classic sign of HE is a sleep disturbance. On a sleep questionnaire, disturbance is seen in $47 \%$ of cirrhotics compared to $4.5 \%$ of controls. ${ }^{37} \mathrm{~A}$ higher frequency of sleep disturbance in cirrhotic patients with MHE has been confirmed in studies using HRQOL questionaires. ${ }^{39,40}$ Sleep disturbance in cirrhosis is not associated with cognitive impairment; thus it may not truly be an MHE symptom. Unsatisfactory sleep is associated with higher scores for depression and anxiety, raising the possibility that the effects of chronic disease may underlie the pathogenesis of sleep disturbance. ${ }^{41}$ Disturbances in cirrhotics may also be related to abnormalities of circadian rhythm. 
Defective memory may be a signe of MHE. Patients with MHE have impaired short- and long-term memory. ${ }^{38}$ This impairment is predominantly related to deficits in attention and visual perception. Memory deficit of MHE seems to comprise short-term but not long-term memory impairment. This can be described as an encoding defect, in which memory recall (or retrieval) is intact.

Several cognitive statements (i.e. complaints), have predictive value for MHE, including impaired psychomotor performance ('I have difficulty doing handwork; I am not working at all'); impaired sleep or rest ('I spend much of the day lying down in order to rest'); decreased attention ('I am confused and start several actions at a time'); and poor memory ('I forget a lot; for example, things that happened recently, where I put things, etc.'). ${ }^{41}$

\section{Health-related quality of life}

\subsection{Effect of MHE on daily functioning}

MHE adversely affects HRQOL. Cognitive impairment in MHE mainly affects complex activities involving attention, information processing and psychomotor skills such as driving a car, planning a trip, etc. whereas basic activities of daily life, such as shopping, dressing, personal hygiene, etc. are preserved. ${ }^{39,42,43}$ Patients with MHE had a significant impairment of daily functioning, such as social interaction, alertness, emotional behavior, sleep, work, home management, recreation and pastimes compared with cirrhotic patients who did not have MHE.39,42 Treatment with lactulose improved both cognitive functions and HRQOL; improvement in the latter was linked to improvement in cognitive function. ${ }^{39}$

\subsection{Effect of MHE on driving}

MHE adversely affects driving skills. Patients with MHE have higher rates of traffic violations and motor vehicle accidents. ${ }^{41}$ Schomerus et al. ${ }^{44}$ were the first to demonstrate a negative effect of psychomotor deficits in patients with MHE on driving fitness. Similar results were reported by Watanabe et al. ${ }^{45}$ Wein et al. ${ }^{46}$ found that the fitness to drive a car was impaired in cirrhotic patients with MHE using a standarizad 90-minute on-road driving test. Increased risk of automobile accidents was related to a decline in cognitive function. ${ }^{4}$ Impairment in attention and speed of mental processing adversely affects an individual's ability to react to unexpected traffic conditions. Patients with MHE have higher rates of traffic violations and motor vehicle accidents. $47-50$ Patients with MHE also had impaired navigation skills. ${ }^{51}$ Navigation, required for safe driving, is a complex process that depends on functioning working memory, attention, and speed of mental processing; impairment in navigation skills correlated with impairment in response inhibition and attention.

\section{Diagnosis of MHE}

The absence of clinical evidence of hepatic encephalopathy is key to the diagnosis of MHE and can only be determined by a detailed assessment of the patient history and a comprehensive neurological assessment of consciousness, cognitive, and motor function.

Various tools have been evaluated for the diagnosis of MHE and include the neuropsychological tests, computerized tests, short neuropsychological and computerized test batteries and neurophysiological tests. Regional cerebral blood flow changes, ${ }^{52}$ and 
magnetic resonance imaging and spectroscopy,53 though useful for understanding pathogenic mechanisms, are currently not considered of diagnostic value.

\subsection{Neuropsychological tests}

Neuropsychological testing is an established methodology for quantifying cognitive impairment due to various forms of encephalopathy, including low-grade or minimal hepatic encephalopathy. Neropsychological tests directly measure cognitive functions that are directly relevant to activities of daily living. They have been applied for the diagnosis of $\mathrm{HE}$ for more than 50 years. In the fifties, measures like the construction or reproduction of a fivepointed star (Fig.1.) or a coil and handwriting have been used for the diagnosis of HE. Someties even more complex figures were presented to the patients to be reproducted. Although all tests were able to depict an increase or decrease of the constructional ability of a patients it was extremely difficult to quantify the test result. In general, psychometric tests have to fulfill the following criteria: they have to be objective, reliable, valid and sensitive. ${ }^{54}$

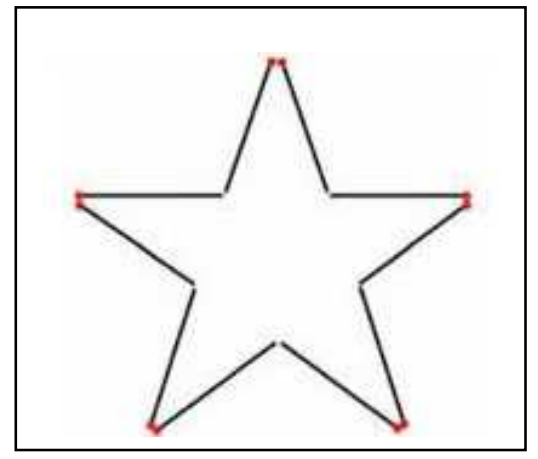

Fig. 1. Star construction test

The neuropsychological features of MHE point to a disorder of executive functioning, particularly selective attention, visuospatial abilities and fine motor skills . ${ }^{4}$ Although these domains are most commonly implicated in MHE, impairments of memory have also been reported. 55,56

The attentional impairments in MHE are observed on a variety of measures. These include measures of cognitive processing speed involving psychomotor responding, such as the Number Connection test A (NCT- A), the Number Connection test B (NCT- B), block design test (BDT), the Digit Symbol test (DST), Line drawing test, Circle dotting, Serial-dotting test (SDOT), Figure connection test. Impairments on measures of cognitive processing speed and response inhibition that do not require a motor response have also been reported (e.g. with verbal fluency tasks and measures such asthe Stroop test). ${ }^{57-60}$ Visuospatial impairments have been primarily reported on block design tasks ${ }^{39,61-63}$ (which also include a motor/practic component), but also on more pure measures of visuospatial perception, such as line orientation or the Hooper test. ${ }^{64,65}$ Fine motor skill impairments have been noted on measures such as the grooved pegboard task, 57,58 and on line tracing tasks (the latter also involve visuospatial abilities). 66,67 
Psychometric test batteries that shall be used for the diagnosis of MHE ought to examine exactly the fields of cognition: visual perception, visuo-spatial orientation, visual construction, concentration, attention and memory. ${ }^{29}$

The small number of neuropsychological tests represent the cerebral disfunction of MHE. This were: the Number Connection test A (NCT- A), the Number Connection test B (NCTB), block design test (BDT), the Digit Symbol test (DST), Line drawing test, Circle dotting. Time-tested with well recognized clinical significance, established.

The number connection test (NCT) is the most widely used test in the psychometric assessment of cirrhotic patients. It was found to be capable of detecting mild mental dysfunction in cirrhotic patients.

The NCT-A (Fig.2) is a test of visuo-spatial orientation and psychomotor speed. The subject is shown a sheet of paper with 25 numbered circles which are randomly spread over the paper. The task is to connect the circles from 1-25 as quick as possible. Test result is the time needed by the subject including error correction time. ${ }^{6}$

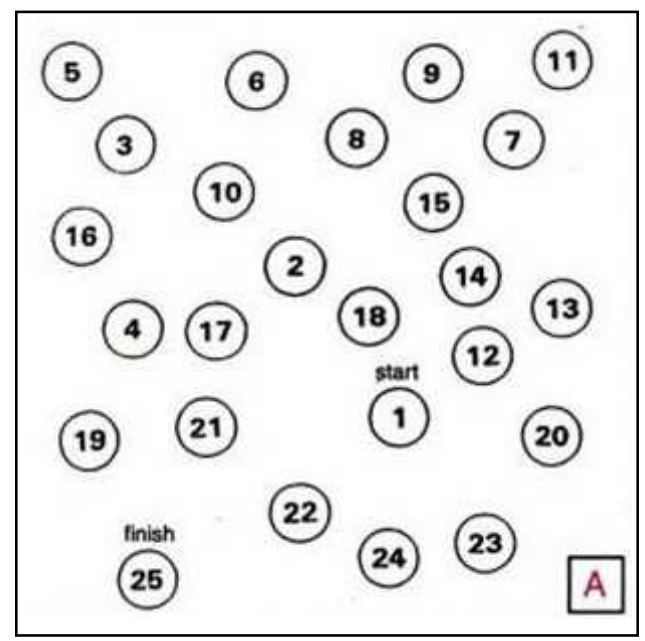

Fig. 2. Number Connection test A (NCT-A) ${ }^{68}$

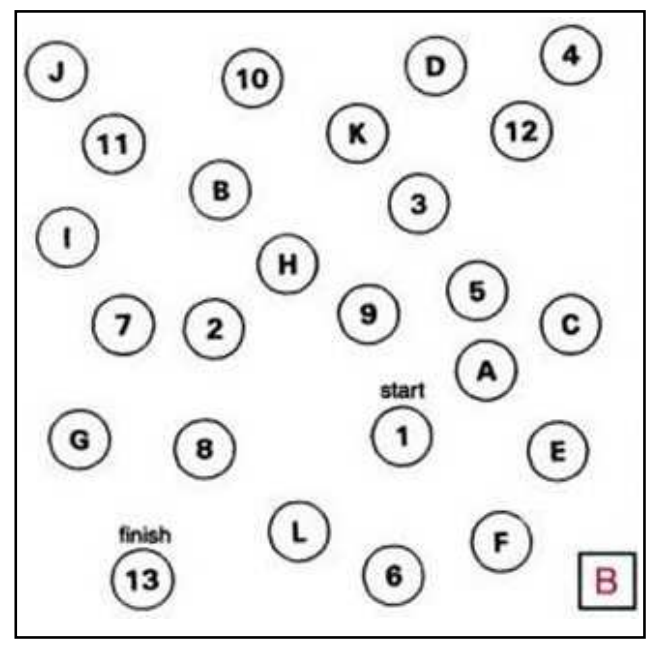

Fig. 3. Number Connection test B (NCT- B $)^{68}$

The NCT-B (Fig.3) is quite similar.The circles include the numbers from 1-13 and the letters from A-L. The subjects are asked to connect numbers and letters in alternating manner, that means go from 1-A-2-B-3-C and so on. Test result is the time needed including error correction time. Besides visuo-spatial orientation and psychomotor speed this test is appropriate to study the ability to shift attention. 68

The Block Design Test (BDT) is a test of visuo-spatial and motor skills (Fig.4). The task is to take 6- 9 blocks that have all white sides, all red sides, and red and white sides and arrange them according to a pattern formed by examiner or shown on a card. Scored for speed and accuracy. 69 


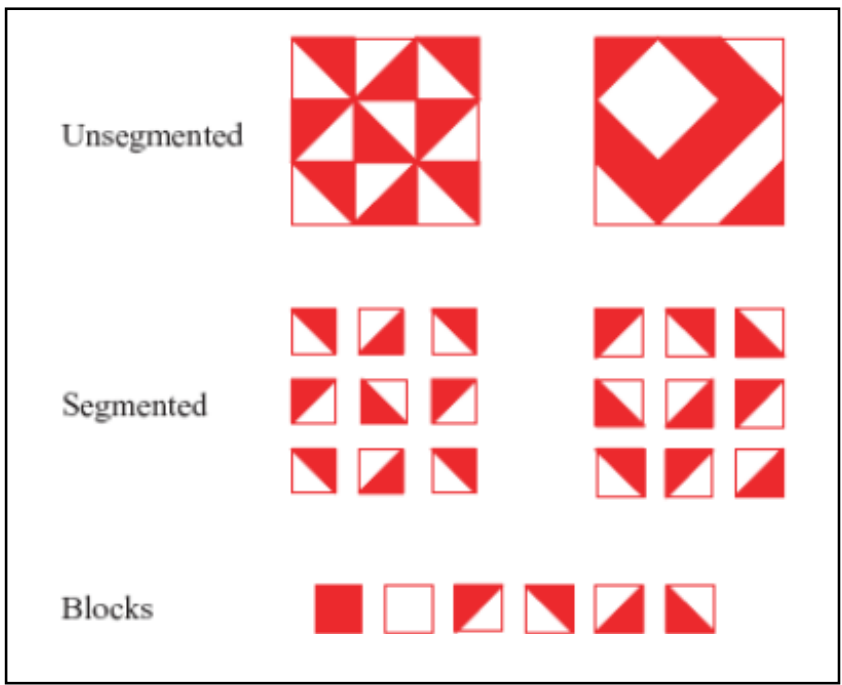

Fig. 4. The Block Design Test (BDT) 69

The Digit Symbol test (DST) (Fig. 5) - the subject is given a series of double-boxes with a number given in the upper part. The task is to draw a symbol pertinent to this number into the lower part of the boxes. Nine fixed pairs of numbers and symbols are given at the top of the test sheet. Test result is the number of boxes correctly filled within 90 seconds. Pathological test results indicate a deficit in visuo-constructive abilities, especially. ${ }^{70}$

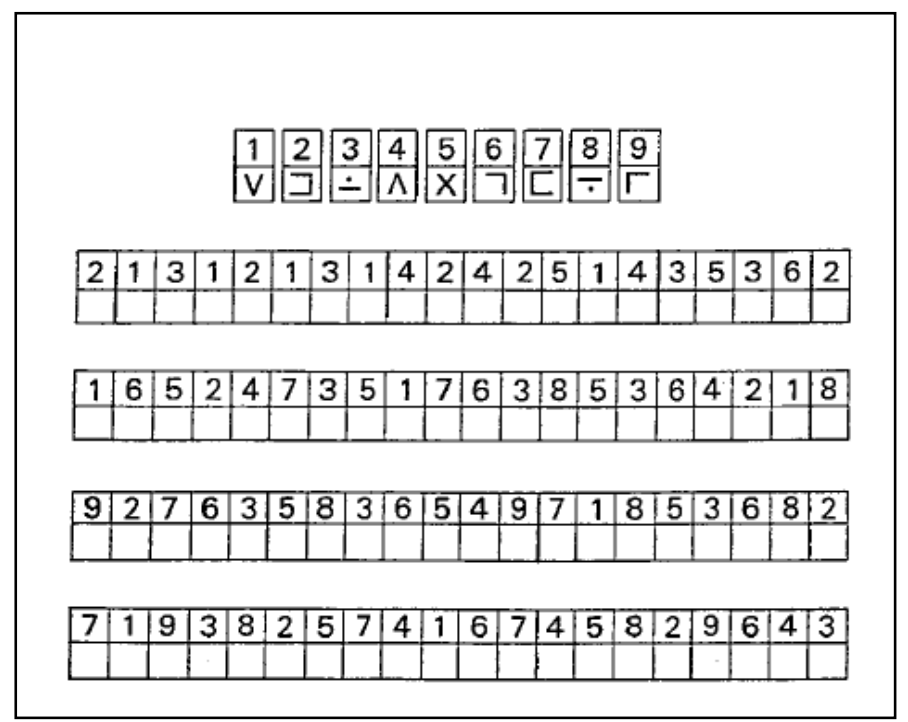

Fig. 5. The Digit Symbol test (DST) ${ }^{70}$ 
The line drawing test (Fig. 6) is a test of motor speed and accuracy. The patients have to follow the route of this labyrinth without crossing or even touching the borderlines. ${ }^{70}$

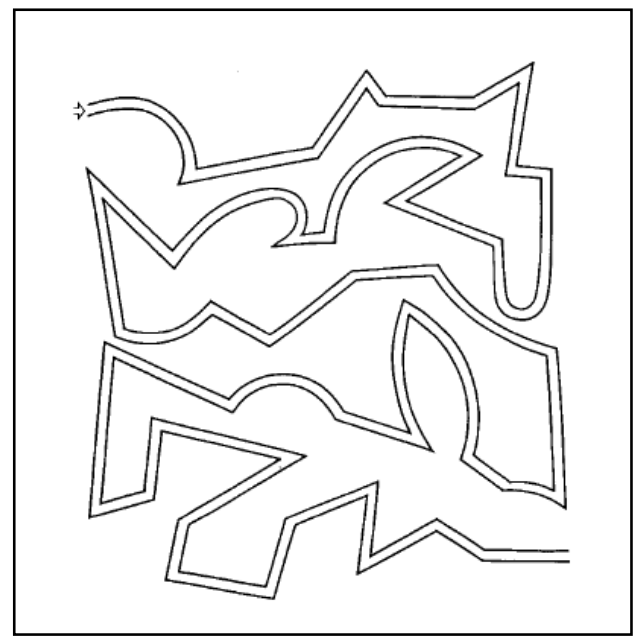

Fig. 6. The line drawing test

For the assessment of the test result the whole route is devided into small sections (Fig.7) and each touching or crossing the border in a section is counted. The number of mistakes and the time needed to go through the labyrinth, both, are test results. ${ }^{70}$

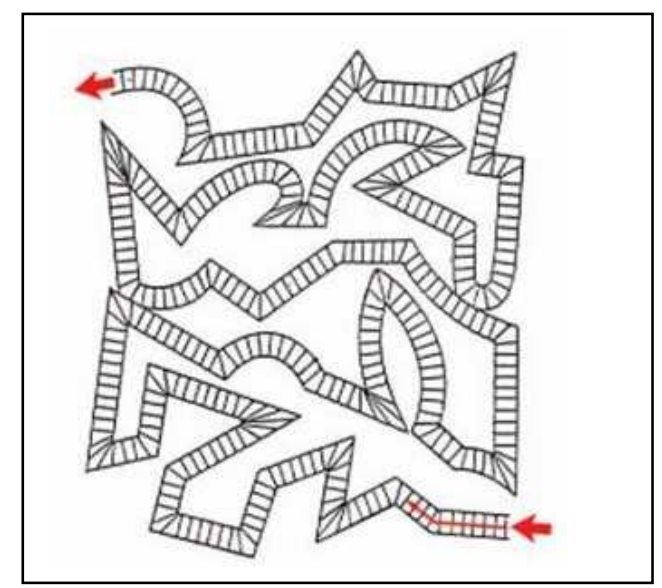

Fig. 7. Division of the whole route of the line drawing test for the assessment of the errors.

The circle dotting test (Fig.8) is the most simple test of the battery. It is a test of pure motor speed. The subjects are asked to put a dot in each of the 100 circles given on the sheet, after they have prepared by dotting the 20 circles at the top of the sheet, first. Test result is the time needed. 


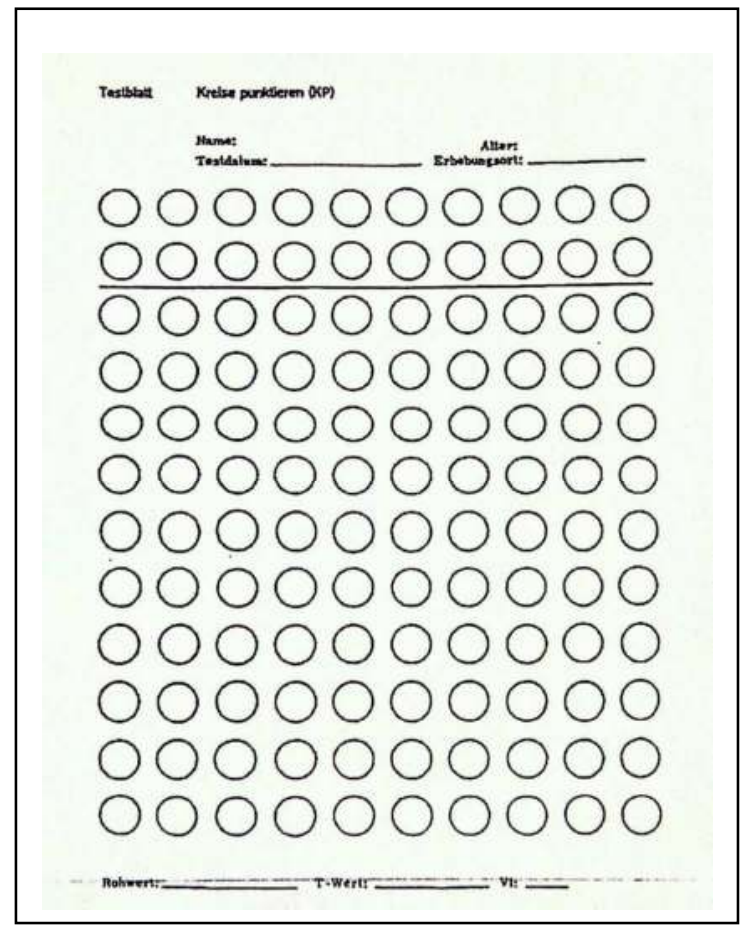

Fig. 8. The circle dotting test

The Working Party recommends that the diagnosis of MHE requires a normal mental status examination and impairment in the performance of at least two of the following tests: NCTA, or figure connection test-A (FCT-A), NCT-B, BDP, DST.41

In 2009, the Commission on Neuropsychological Assessment of Hepatic Encephalopathy concluded that neuropsychological test batteries aimed at measuring multiple domains of cognitive function are generally more reliable than single tests, and tend to be more strongly correlated with functional status. ${ }^{71}$ Both the Repeatable Battery for the Assessment of Neuropsychological Status (RBANS) ${ }^{72}$ and PSE-Syndrom-Test $7^{73}$ may be used for this purpose.

The PSE-Syndrom-Test, developed in Germany and validated in several other European countries, incorporates older assessment tools such as NCT-A and NCT-B, the line-tracing test (LTT), the serial-dotting test (SDT), and DST.

The psychometric hepatic encephalopathy score (PHES) is a standardized test battery including NCT-A and B, the line-tracing test for time $(t)$ and error $(e)$, the serial-dotting test, and the digit symbol test. This battery examines many of the abnormalities seen in patients with MHE, including motor speed and accuracy, visuo-spatial orientation, visual perception, visual construction, attention, concentration, and, to a lesser extent, memory. PHES has a prognostic value for the occurrence of overt HE and mortality in cirrhotic patients. ${ }^{74,75}$ 
The RBANS contains measures of verbal and visual anterograde memory, working memory, cognitive processing speed, language (including semantic fluency) and visuospatial function (line orientation and figure copy). It is a portable pencil-and-paper test that requires a

folding stimulus booklet and paper record form to administer. Administration time is approximately 20-25 min. ${ }^{71}$ In the study of Sorrel et al. ${ }^{76}$ RBANS scores were strongly correlated with liver disease as measured by the model for end-stage liver disease staging. Scores on the RBANS also predicted disability independently of liver disease severity in this study.

Use of either the RBANS or the PSE-Syndrom-Test is recommended for diagnosing and monitoring minimal hepatic encephalopathy. The choice of which battery to use should be based upon the availability of local translations and normative data. ${ }^{71}$

\subsection{Neurophysiological tests}

Quantitative neurophysiologic tools include Simple electroencephalography (EEG), evoked potentials (auditory, visual, Somatosensory) and P300 (type of auditory evoked potential).

Changes in EEG/evoked responses are non-specific.

The major finding on EEG is a general decrease in wave frequency and an increase in wave amplitude. First, socalled theta-waves with a frequency between 4 and 7 cps occur, then these theta waves predominate and are committed by delta waves with a frequency of 1-3 cps. Preterminally there is a loss of wave amplitude and a flattening of the curve. These abnormalities may be found even in cirrhotics without clinical signs of encephalopathy. There is no close correlation between the grade of $\mathrm{HE}$ and the degree of EEG abnormalities. ${ }^{70}$ The sensitivity of the EEG for the diagnosis of subclinical HE is limited compared to psychometric tests. ${ }^{70}$ The EEG is useful for follow-up examinations, predominantly.

Among EEG variations, the most sensitive test is computer-assisted analysis, including the mean dominant EEG frequency and the power of a particular rhythm.77,78 Quantified-EEG has a prognostic value for occurrence of bouts of overt HE and mortality in cirrhotic patients. ${ }^{78}$

Evoked potentials are subdivided into the group of exogenous evoked potentials and endogenous evoked potentials. The exogenous evoked potentials like the flash or checkerboard visual evoked potentials (VEP), brainstem auditory evoked potentials (BAEP) and somatosensory evoked potentials are used to examine the function of sensory pathways. The endogenous evoked potentials are measures of cognitive function. In the only study that compares the different exogenous evoked potentials for their diagnostic ensitivity in hepatic encephalopathy, the BAEP were the most sensitive measure for the diagnosis of HE. ${ }^{79}$

Among evoked responses, the P300 peak obtained in an auditory oddball paradigm is the most sensitive test. ${ }^{80-83}$ These tests can supplement neurological or neuropsychiatric examination. It has been demonstrated that there was a greater likelihood of development of overt HE in cirrhotic patients with abnormal P300 event-related potential latencies and NCT than in patients with no such abnormality. ${ }^{80}$ 
Neurophysiological tests can be used during follow up to demonstrate change in a patient's condition. Their major limitations are: (i) need for specialized equipment and technical expertise for evaluation and interpretation; and (ii) inability to perform these tests in an outpatient clinic. ${ }^{41}$

The changes observed in cerebral blood flow and metabolism in SPET, PET, and ${ }^{1} \mathrm{H}$ and ${ }^{31} \mathrm{P}$ MRS studies reflect the pathogenic process that underlies the condition rather than providing diagnostic information. Similarly, the morphological brain abnormalities identified in this population, including mild brain oedema, hyperintensity of the globus pallidus and other subcortical nuclei observed in cerebral MR studies, and the central and cortical atrophy observed in neural imaging studies, are unlikely to have diagnostic utility. 4

\subsection{Computerized tests}

Computerized psychometric tests measuring both the reaction time and the accuracy of performing tasks requiring psychomotor speed, attention, short-term memory, or choice ability.

Critical flicker frequency (CFF) tests the ability of a patient to perceive flickering and its fusion threshold. The CFF threshold measures visual discrimination and general arousal. ${ }^{84}$ CFF is a simple, reliable and accurate method for the diagnosis of MHE. The technique shows little dependence on age, education or training. 75,85

Inhibitory control test (ICT) is a computerized test of attention and response inhibition that has been used to characterize attention deficit disorder, schizophrenia and traumatic brain injury. ICT has been validated for the diagnosis of MHE in USA and found to be reliable and sensitive for detection as well as follow-up of patients with MHE. 86

\subsection{Magnetic resonance imaging and spectroscopy}

Magnetic resonance imaging (MRI) identified the morphological brain abnormalities in the population of patients with cirrhosis, including mild brain oedema, hyperintensity of the globus pallidus and other subcortical nuclei, and central and cortical atrophy. High-signal abnormalities on T1-weighted images in the globus pallidum have been observed in cirrhotic patients, even without clinical evidence of HE. Deposition of manganese is regarded as the most likely explanation of this high-signal abnormality. ${ }^{87}$ There is no direct correlation between pallidal hyperintensity and grade of encephalopathy. ${ }^{8}$ Basal ganglia T1-weighted signal intensity and manganese accumulation appear to be related to the underlying degree of portal-systemic shunting rather than directly to neuropsychiatric impairment. ${ }^{90}$ Hyperintense globus pallidus on MRI is common in patients with liver cirrhosis and also occurs in patients with noncirrhotic portal hypertension.

Magnetic resonance spectroscopy (MRS) shows a decrease in myo-inositol/creatine and choline/creatine ratios in the white matter with an increase in the Glx (glutamine and glutamate) concentration in the basal ganglia in patients with MHE.91,92 Liver transplantation as well as lactulose therapy have been shown to reverse these changes at 4 weeks and later after transplantation. ${ }^{91}$ However, the ability of MRS to differentiate between cirrhotic patients without HE and those with MHE has not been conclusively shown. ${ }^{41}$

Diffusion-weighted imaging allows assessment of intracellular and extracellular water content in the brain, which helps in differentiating cytotoxic from vasogenic edema. ${ }^{93}$ 
Diffusion tensor imaging has revealed that mean diffusivity, a measure of water movement across cell membranes, is significantly higher in patients with MHE in the regions of the corpus callosum, internal capsules, caudate nuclei and occipital white matter. Increase in mean diffusivity indicates the presence of interstitial brain edema. Mean diffusivity values increase as the grade of HE increases, suggesting that brain edema present in patients with HE may contribute to its pathogenesis. ${ }^{94}$ Mean diffusivity values decreased significantly and there was a corresponding improvement in neuropsychological test scores in patients with MHE after three weeks of lactulose therapy. ${ }^{94} \mathrm{MR}$ imaging techniques therefore complement neuropsychological evaluation of MHE. 41

\section{Diagnostic criteria for MHE}

The diagnostic criteria for MHE have not been standardized but rest on careful patient history and physical examination, normal mental status examination, demonstration of abnormalities in cognition and/or neurophysiological function, and exclusion of concomitant neurological disorders.

No consensus on diagnostic criteria or diagnostic tests has been established.

- Cirrhotic patients

- Without clinical signs of encephalopathy

- Perform worse in psychometric tests when compared with healthy controls

- Affects an estimated $60 \%(50 \% \text { to } 80 \%)^{*}$ of patients with cirrhosis

- Cerebral dysfunction has a major impact on patients' daily living

- The presence of a disease that can cause MHE, such as, cirrhosis and/or the presence of a portalsystemic shunt

- normal mental status on clinical examination

- demonstration of abnormalities of cognition and/or neurophysiological variables

- exclusion of concomitant neurological disorders.

The INASL Working Party recommends that all patients with cirrhosis be screened for the presence of MHE using a standard battery of psychometric tests, PHES, CFF or ICT, depending upon the availability of tests and their validation for local populations from different parts of the world (Fig. 1). Patients whose index psychometric or computerized test results do not indicate pathology should be screened every 6-12 months.

It has been shown conclusively that cognitive functions improve with therapy for MHE.3,62-67 Such therapy may improve HRQOL of patients with MHE3,67 and delay the development of HE.68 Hence all patients with liver cirrhosis should be subjected to testing for MHE. Special attention should be given to those who have cognitive symptoms and high-risk groups such as active drivers, patients handling heavy machines or reporting decline in work performance.

\section{Natural history}

\subsection{Development of overt hepatic encephalopathy}

Patients with MHE may improve, remain unchanged or deteriorate and develop overt HE over a long-term follow-up. 
The frequency of MHE increases as the severity of liver disease increases.4,13-16,18,22 In view of a high frequency of MHE in patients with liver disease, it is important to understand its impact on future clinical outcomes, such as occurrence of overt HE, quality of life and survival, and to determine whether treatment of MHE can induce improvements in these outcomes.

Several studies that looked at the frequency of development of overt HE in cirrhotic patients found that those with MHE developed overt HE more often during follow up than those without MHE (Table 4).4,15,17,20,48,88,89 In addition, some studies have shown an increased risk of death in patients with liver cirrhosis and MHE compared to those without MHE (Table 4).20,22,88 However, patients with MHE had poorer liver function than those without MHE in these studies, making it difficult to ascribe the poor outcome to the presence of MHE. Das et al.4 studied the relationship of progression of MHE to overt HE in relation to the severity of liver dysfunction and found that the rate of progression to overt HE was much higher in patients with MHE and a CTP score $>6$ than in those with MHE and a CTP score _ 6. Amodio et al.88 found that the presence of MHE and that of liver dysfunction were both associated with mortality on univariate analysis; however, on multivariate analysis, liver functional status was the only independent predictor of mortality. In another study, progression of MHE to overt HE was associated with abnormal response to oral glutamine challenge, which in turn was associated with poor liver function.90 Furthermore, MHE in patients with preserved liver function but large portalsystemic shunts (congenital shunts, non-cirrhotic portal hypertension and cirrhosis with preserved liver function) appears to have a good outcome, even though these data are based on a small number of patients.10 Thus, it appears that the higher risk of overt HE or death in patients with MHE may not be related to MHE per se but to the poorer liver function in patients with MHE.

\section{Survival}

Current data suggest that patients with MHE tend to have more frequent episodes of overt $\mathrm{HE}$ and poorer survival than in those without MHE, and indicate that patients with MHE have a more advanced liver disease. Child-Turcotte-Pugh score and PHES were associated with a poor prognosis

\section{Treatment}

Ammonia plays a key role in the pathogenesis of MHE Empiric therapy is based on the principle of reducing the production and absorption of ammonia in the gut-a number of agents are beneficial for this purpose.

\subsection{Nutritional interventions}

The European Society for Parenteral and Enteral Nutrition recommended, in 2006, that patients with cirrhosis must eat at least $1.2 \mathrm{~g} / \mathrm{kg}$ of protein daily. They also recommended that the diet of patients with cirrhosis should be supplemented with branched-chain amino acids (BCAAs) and vegetable protein once HE has developed. Vegetable-based protein is better tolerated by patients with cirrhosis than meat-based protein . 


\subsection{Pharmacological therapy}

Non-absorbable disaccharides include lactulose and lactitol. Treatment for MHE may be initiated with lactulose; patients should receive $30-60 \mathrm{~mL}$ of lactulose in two or three divided doses so that they pass two to three semi-soft stools per day. Although the appropriate duration of therapy for MHE is unsettled, at least three studies suggest that treatment may be advised for 3-6 months. $(3,67,95)$

Lactulose decreases blood ammonia levels, and improves psychometric performance and HRQOL (Table 5).3,59,62,64,67,91-95 Using cerebral diffusion tensor imaging, Kale et al.59 showed that interstitial brain edema observed in patients with MHE resolves after treatment for 3 weeks with lactulose in parallel with improvements in neuropsychiatric performance.

Prasad et al.3 studied the effect of treatment of MHE with lactulose on psychometric performance (measured by NCT, FCT-A, FCT-B, picture completion and block-design tests) and HRQOL (measured by Sickness Impact Profile [SIP]). Patients with MHE showed significant impairment in 11 scales of the SIP, the psychosocial and physical subscores, and in the total SIP. Patients received $30-60 \mathrm{~mL}$ of lactulose in two or three divided doses so that the patient passed two to three semi-soft stools per day. Following lactulose therapy for 3 months, both psychometric performance and HRQOL improved; MHE reversed in $64.5 \%$ of treated patients compared with $6.7 \%$ in the no-treatment group $(P<0.0001)$. Significant improvement was found in five (emotional behavior, ambulation, mobility, sleep/rest and recreation and pastimes) of the 12 scales of the SIP and in the total psychosocial and physical sub-scores in the treated patients compared with the untreated patients. Improvement in HRQOL was linked to improvement in cognitive function. A recent study that compared lactulose, a probiotic and LOLA with no treatment, confirmed these findings.67 Lactulose or lactitol, both non-absorbable, synthetic disaccharides with multiple effects on gut flora, are regarded as intestinal prebiotics.96 Dietary addition of lactulose can exert a bifidogenic effect accompanied by a favorable effect on colonic $\mathrm{NH}_{3}$ metabolism.97 Ameta-analysis of randomized trials of lactulose versus placebo or no intervention in treatment of patients with MHE showed that the treatment with lactulose was associated with improvement in psychometric (cognitive) performance. 35

Branched-chain amino acids, flumazenil, L-ornithine L-aspartate, acetyl L-carnitine, and probiotics/synbiotics. A majority of these attempts were aimed at reducing blood ammonia level, and most studies have shown improvement in psychometric measurements, ammonia levels, cerebral edema and health-related quality of life (HRQoL)

\subsection{Prebiotics, probiotics or synbiotics}

Prebiotics, probiotics or synbiotics (probiotics and fermentable fiber) are effective in treating patients with MHE,63-67 and can also be used as long-term therapy. Liu et al.65 showed that modulation of gut microecology and acidification of gut lumen in patients with liver cirrhosis and MHE by treatment with synbiotics resulted in increased fecal content of nonurease-producing Lactobacillus species, whereas the number of urease-producing pathogenic Escherichia coli and Staphylococcal species decreased. This effect persisted for 14 days after cessation of supplementation. It was associated with a significant reduction in blood ammonia and endotoxin levels and reversal ofMHEin nearly $50 \%$ of the patients. The severity of liver disease, as assessed according to CTP class, also improved in nearly $50 \%$ of 
the patients. In a recent randomized control trial, supplementation with probiotic yogurt resulted in a significant reversal ofMHEin the group receiving yogurt compared

to no treatment.63 Treatment with a probiotic preparation also improves HROQL.67 Prebiotics, probiotics or synbiotics are efficacious in the treatment of HE by decreasing bacterial urease activity, $\mathrm{pH}$ in the gut lumen, ammonia absorption and total ammonia in the portal blood, and by improving nutritional status of gut epithelium resulting in decreasing intestinal permeability. In addition, they help ameliorate the inflammation and oxidative stress in the hepatocytes, leading to increased hepatic clearance of ammonia.98 These mechanisms may be additive or synergistic in treating MHE. Probiotics may represent a safe, effective, long-term therapy for MHE and may be an alternative to lactulose.

\subsection{L-ornithine-L-aspartate}

Clinical studies evaluating the role of LOLA in the treatment of MHE did not show its effectiveness; however, these studies were small and underpowered. A recent study that compared lactulose, a probiotic and LOLA with no treatment, however, showed that LOLA is as effective as lactulose or a probiotic preparation in improving psychometric performance and HRQOL.67 Larger prospective studies are warranted to evaluate the role of LOLA before it can be recommended for the treatment of MHE.

\subsection{Antibiotics}

The role of antibiotics in MHE has not been evaluated. Prospective studies with poorly absorbed antibiotics are required to evaluate their efficacy in improving MHE.

Antibiotics: Rifaximin, Neomycin

\section{References}

[1] Häussinger D, Blei AT.Portal hypertension and its complications. In : Rodes J, Benhamou JP, Blei AT, Reichen J, Rizzetto M, eds. Textbook of hepatology. From basic science to clinical practice, third edition. Oxford: Blackwell Publishing ; 2007: 623-760

[2] Dhiman RK, Chawla YK. Minimal hepatic encephalopathy. Indian J Gastroenterol 2009; 28:5-16

[3] Prasad S, Dhiman RK, Duseja A, Chawla YK, Sharma A, Agarwal R. Lactulose improves cognitive functions and health-related quality of life in patients with cirrhosis who have minimal hepatic encephalopathy. Hepatology 2007; 45: 549-59

[4] Tan HH, Lee GH, Thia KTJ, Ng HS, Chow WC, Lui HF. Minimal hepatic encephalopathy runs a fluctuating course: results from a three-year prospective cohort follow-up study. Singapore Med J 2009; 50(3): 255-260

[5] Amodio P, Montagnese S, Gatta A , Morgan MY. Characteristics of minimal hepatic encephalopathy. Metabolic Brain Disease 2004; 19: 253-267

[6] Arguedas MR, DeLawrence TG, McGuire BM. Influence of hepatic encephalopathy on health-related quality of life in patients with cirrhosis. Dig Dis Sci 2003; 48: 16221626

[7] Mullen KD. Review of the final report of the 1998 Working Party on definition, nomenclature and diagnosis of hepatic encephalopathy. Aliment Pharmacol Ther 2007;25:11-16 
[8] Kharbanda PS, Saraswat VA, Dhiman RK. Minimal hepatic encephalopathy: diagnosis by neurophchological and neurophysiological methods. Indian J Gastroenterol 2003; 22: 537-541

[9] Ortiz M, Jacas C, Cordoba J. Minimal hepatic encephalopathy: diagnosis, clinical signifi cance and recommendations. J Hepatol 2005;42: 45-53

[10] Lockwood AH. "What's in a name?" Improving the care ofcirrhotics. J Hepatol 2000; 32: 859-861

[11] Quero Guillén JC, Groeneweg M, Jiménez Sáenz M, Schalm SW, Herrerías Gutiérrez JM. Is it medical error if we do not screen cirrhotic patients for minimal hepatic encephalopathy? Rev Esp Enferm Dig 2002; 94: 544-557

[12] Amodio P, Del Piccolo F, Petteno E, et al. Prevalence and prognostic value of quantified electroencephalogram (EEG) alterations in cirrhotic patients. J Hepatol 2001; 35: 37-45

[13] Boyer TD, Haskal ZJ, American Association for the Study of Liver Diseases. The role of transjugular intrahepatic portosystemic shunt in the management of portal hypertension. Hepatology 2005; 41: 386-400

[14] Kurmi R, Reddy K, Dhiman RK, et al. Psychometric hepatic encephalopathy score, critical flicker frequency and p300 event-related potential for the diagnosis of minimal hepatic encephalopathy: Evidence that psychometric hepatic encephalopathy score is enough. Indian J Gastroenterol 2008; 27: S1

[15] Butterworth RF. Pathophysiology of hepatic encephalopathy: a new look at ammonia. Metab Brain Dis 2002; 17: 221-227

[16] Vaquero J, Chung C, Blei AT. Brain edema in acute liver failure. A window to the pathogenesis of hepatic encephalopathy. Ann Hepatol 2003; 2: 12-22

[17] Takano T, Tian GF, Peng W, et al. Astrocyte-mediated control of cerebral blood fl ow. Nat Neurosci 2006; 9: 260-270

[18] Ahboucha S, Butterworth RF. The neurosteroid system: implication in the pathophysiology of hepatic encephalopathy. Neurochem Int 2008; 52: 575-587

[19] Sharma P, Sharma BC, Puri V, Sarin SK. Minimal hepatic encephalopathy in patients with extrahepatic portal vein obstruction. Am J Gastroenterol 2008;103: 1406-1412

[20] Balata S, Damink SW, Ferguson K, et al. Induced hyperammonemia alters neuropsychology, brain MR spectroscopy and magnetization transfer in cirrhosis. Hepatology 2003; 37: 931-939

[21] Cordoba J, Alonso J, Rovira A, et al. The development of low-grade cerebral edema in cirrhosis is supported by the evolution of (1)H-magnetic resonance abnormalities after liver transplantation. J Hepatol 2001; 35: 598-604

[22] Lockwood AH, Yap EW, Wong WH. Cerebral ammonia metabolism in patients with severe liver disease and minimal HE. J Cereb Blood Flow Metab 1991; 11:337-341

[23] Kale RA, Gupta RK, Saraswat VA, et al. Demonstration of interstitial cerebral edema with diffusion tensor MR imaging in type $C$ hepatic encephalopathy. Hepatology 2006; 43: 698-706

[24] Chung RT, Podolsky DK2005. Cirrhosis and its complications. In Kasper DL, Braunwald E, Fauci AS,eds. Harrison's Principles of Internal Medicine, 16th edition. New York, NY: McGraw-Hill;2005: 1858-69

[25] Lozeva-Thomas V. Serotonin brain circuits with a focus on hepatic encephalopathy. Metab Brain Dis 2004; 19:413-20

[26] Capocaccia L, Cangiano C, Cascino A, Calcaterra V,Cardelli P, Rossi FF. Influence of phenylethanolamine on octopamine plasma determination in hepatic encephalopathy. Clin Chim Acta 1979; 93:371-6. 
[27] Fischer JE, Rosen HM, Ebeid AM, James JH, Keane JM,Soeters PB. The effect of normalization of plasma amino acids on hepatic encephalopathy in man. Surgery 1976;80:77-91.

[28] Yoshida Y, Higashi T, Nouso K, Nakatsukasa H, Nakamura SI, Watanabe A, et al. Effects of zinc deficiency/zinc supplementation on ammonia metabolism in patients with decompensated liver cirrhosis. Acta Med Okayama 2001; 55:349-55.

[29] Marchesini G, Fabbri A, Bianchi G, Brizi M, Zoli M. Zinc supplementation and amino acid-nitrogen metabolism in patients with advanced cirrhosis. Hepatology 1996; 23:1084-92.

[30] Cash WJ, McConville P, McDermott E, McCormick PA, Callender ME, McDougall NI. Current concepts in the assessment and treatment of hepatic encephalopathy. QJM 2010;103: 9-16

[31] Das K, Singh P, Chawla Y, Duseja A, Dhiman RK, Suri S. Magnetic resonance imaging of brain in patients with cirrhotic and non-cirrhotic portal hypertension. Dig Dis Sci 2008; 53: 2793-2798

[32] Rama Rao KV, Reddy PV, Hazell AS, Norenberg MD. Manganese induces cell swelling in cultured astrocytes. Neurotoxicology 2007; 28:807-812

[33] Sundaram V, Shaikh OS. Hepatic encephalopathy: pathophysiology and emerging therapies. Med Clin North Am 2009;3: 819-36

[34] Shawcross DL, Wright G, Olde Damink SW, Jalan R. Role of ammonia and inflammation in minimal hepatic encephalopathy. Metab Brain Dis 2007; 22: 125138

[35] Shawcross DL, Davies NA, Williams R, Jalan R. Systemic inflammatory response exacerbates the neuropsychological effects of induced hyperammonemia in cirrhosis. J Hepatol 2004; 40: 247-254.

[36] Zhao HY, Wang HJ, Zhi LU, Zhen XU. Intestinal microflora in patients with liver cirrhosis. Chin. J. Dig. 2004; 5: 64-7.

[37] Liu Q, Duon ZP, Ha DK et al. Synbiotic modulation of gut flora:effect on minimal hepatic encephalopathy in patients with cirrhosis.Heptology 2004; 39: 1441-9.

[38] Cordoba J, Cabrera J, Lataif L, Pener P, Zee P, Blei AT. High prevalence of sleep disturbances in cirrhosis. Hepatology 1998; 27: 339-45.

[39] Weissenborn K, Heidenreich S, Giewekemeyer K, Ruckert N, Hecker H. Memory function in early hepatic encephalopathy. J.Hepatol. 2003; 39: 320-5.

[40] Prasad S, Dhiman RK, Duseja A, Chawla YK, Sharma A, Agarwal R. Lactulose improves cognitive functions and health-related quality of life in patients with cirrhosis who have minimal hepatic encephalopathy. Hepatology 2007; 45: 549-59.

[41] Goeneweg M, Moerland W, Quero JC. Screening of subclinical hepatic encephalopathy. J. Hepatology 2000; 32: 748-53.

[42] Dhiman R, Saraswat V, Sharma B, et al. Minimal hepatic encephalopathy: Consensus statement of a working party of Indian National Association for study of the liver. Journal of Gastroenterology and Hepatology 2010;25: 1029-41.

[43] Groeneweg M, Quero JC, De Bruijn I et al. Subclinical hepatic encephalopathy impairs daily functioning. Hepatology 1998; 28:45-9.

[44] Schomerus H, Hamster W. Quality of life in cirrhotics with minimal hepatic encephalopathy. Metab. Brain. Dis. 2001; 16:37-41.

[45] Schomerus H, Hamster W, Blunck H, Reinhard U, Mayer K, Dolle W. Latent portasystemic encephalopathy. I. Nature of cerebral functional defects and their effect on fitness to drive. Dig. Dis. Sci.1981; 26: 622-30. 
[46] Watanabe A, Tuchida T, Yata Y, Kuwabara Y. Evaluation of neuropsychological function in patients with liver cirrhosis with special reference to their driving ability. Metab. Brain. Dis. 1995;10: 239-48.

[47] Wein C, Koch H, Popp B, Oehler G, Schauder P. Minimal hepatic encephalopathy impairs fitness to drive. Hepatology 2004; 39:739-45.

[48] Marotolli RA, Cooney LM, Wagner S, Doucette J, Tinetti ME. Predictors of automobile crashes and moving violations among elderly drivers. Ann. Intern. Med. 1994; 121: 842-6.

[49] Bajaj JS, Hafeezullah M, Hoffmann RG, Saeian K. Minimal hepatic encephalopathy: a vehicle for accidents and traffic violations. Am. J. Gastroenterol. 2007; 102: 1903-9.

[50] Bajaj JS, Ananthakrishnan AN, McGinley EL, Hoffmann RG, Brasel KJ. Deleterious effect of cirrhosis on outcomes after motor vehicle crashes using the nationwide inpatient sample. Am. J. Gastroenterol. 2008; 103: 1674-81.

[51] Bajaj JS, Saeian K, Schubert CM et al. Minimal hepaticencephalopathy is associated with motor vehicle crashes: the reality beyond the driving test. Hepatology 2009; 50: 1175-83.

[52] Bajaj JS, Hafeezullah M, Hoffmann RG et al. Navigation skill impairment: Another dimension of the driving difficulties in minimal hepatic encephalopathy. Hepatology 2008; 47: 596-604.

[53] Venktaramarao SH, Mittal, Prabhakar S, Dhiman RK. Brain perfusion single photon emission computed tomography (SPECT) abnormalities in patients with minimal hepatic encephalopathy (abstract). J Gastroenterol Hepatol 2008; 23 : A62

[54] Grover VP, Dresner MA, Forton DM, et al. Current and future applications of magnetic resonance imaging and spectroscopy of the brain in hepatic encephalopathy. World J Gastroenterol 2006; 12: 2969-2978.

[55] Weissenborn K. Diagnosis of subclinical hepatic encephalopathy. Med Sci Monit 1999; 5: 568-575.

[56] Bahceci F, Yildirim B, Karincaoglu M, Dogan I, Sipahi B. Memory impairment in patients with cirrhosis. J Natl Med Assoc 2005; 97:213-6.

[57] Meyer T, Eshelman A, Abouljoud M. Neuropsychological changes in a large sample of liver transplant candidates. Transplant Proc 2006; 38: 3559-60.

[58] McCrea M, Cordoba J, Vessey G, Blei AT, Randolph C. Neuropsychological characterization and detection of subclinical hepatic encephalopathy. Arch Neurol 1996; 53: 758-63.

[59] Tarter RE, Van Thiel DH, Arria AM, Carra J, Moss H. Impact of cirrhosis on the neuropsychological test performance of alcoholics. Alcohol Clin Exp Res 1988; 12: 619-21.

[60] Binesh N, Huda A, Thomas MA, et al. Hepatic encephalopathy: a neurochemical, neuroanatomical, and neuropsychological study. J Appl Clin Med Phys 2006; 7: 8696.

[61] Mattarozzi K, Stracciari A, Vignatelli L, et al. Minimal hepatic encephalopathy: longitudinal effects of liver transplantation. Arch Neurol 2004; 61: 242-7.

[62] Gitlin N, Lewis DC, Hinkley L. The diagnosis and prevalence of subclinical hepatic encephalopathy in apparently healthy, ambulant, non-shunted patients with cirrhosis. J Hepatol 1986; 3: 75-82.

[63] Sood GK, Sarin SK, Mahaptra J, Broor SL. Comparative efficacy of psychometric tests in detection of subclinical hepatic encephalopathy in nonalcoholic cirrhotics: search for a rational approach. Am J Gastroenterol 1989; 84: 156-9. 
[64] Tarter RE, Hegedus AM, Van Thiel DH, et al. Nonalcoholic cirrhosis associated with neuropsychological dysfunction in the absence of overt evidence of hepatic encephalopathy. Gastroenterology 1984; 86: 1421-7.

[65] Binesh N, Huda A, Thomas MA, et al. Hepatic encephalopathy: a neurochemical, neuroanatomical, and neuropsychological study. J Appl Clin Med Phys 2006; 7: 86-96.

[66] Mittal VV, Sharma P, Sharma BC, Sarin S. Treatment of minimal hepatic encephalopathy: A randomised controlled trial comparing lactulose, probiotics and 1-ornithine l-aspartate with placebo. Hepatology 2009; 50: 471A

[67] Minguez B, Garcia-Pagan JC, Bosch J, et al. Noncirrhotic portal vein thrombosis exhibits neuropsychological and MR changes consistent with minimal hepatic encephalopathy. Hepatology 2006;43: 707-14.

[68] Schomerus H, Hamster W. Neuropsychological aspects of portalsystemic encephalopathy. Metab Brain Dis 1998; 13: 361-77.

[69] Kugler CF, Petter J, Taghavy A, et al. Dynamics of cognitive brain dysfunction in patients with cirrhotic liver disease: an event-related P300 potential perspective. Electroencephalogr Clin Neurophysiol 1994; 91: 33-41.

[70] Conn HO: Trailmaking and Number Connection Tests in the Assessment of Mental State in Portal Systemic Encephalopathy. Dig Dis, 1977; 22(6): 541-50.

[71] Loguercio C, Del Vecchio-Blanco C, Coltorti M: Psychometric Tests and Latent PortalSystemic Encephalopathy. Brit J Clin Pract 1984; 38: 407-11.

[72] Weissenborn K. Diagnosis of subclinical hepatic encephapolaphy. Med Sci Monit 1999; 5:568-575.

[73] Randolph C, Hilsabeck R, Kato A, et al. Neuropsychological assessment of hepatic encephalopathy: ISHEN practice guidelines. Liver International 2009;629-35.

[74] Randolph C. The Repeatable Battery for the Assessment of Neuropsychological Status (RBANS). San Antonio: The Psychological Corporation, 1998.

[75] Schomerus H, Weissenborn K, Hamster W, Ruckert N, Hecker H. PSE-Syndrom-Test. Frankfurt: Swets \& Zeitlinger B.V., 1999.

[76] Dhiman RK, Kurmi R, Thumburu KK et al. Diagnosis and prognostic significance of minimal hepatic encephalopathy in patients with cirrhosis of liver. Dig. Dis. Sci. 2010; 55:2381-90.

[77] Romero-Gómez M, Córdoba J, Jover R et al. Value of the critical flicker frequency in patients with minimal hepatic encephalopathy.Hepatology 2007; 45: 879-85.

[78] Sorrell JH, Zolnikov BJ, Sharma A, Jinnai I. Cognitive impairment in people diagnosed with end-stage liver disease evaluated for liver transplantation. Psychiatry Clin Neurosci 2006; 60: 174-81.

[79] Amodio P, Quero JC, Del Piccolo F, Gatta A, Schalm SW. Diagnostic tools for the detection of subclinical hepatic encephalopathy: comparison of standard and computerized psychometric tests with spectral-EEG. Metab. Brain. Dis. 1996; 11:315-27.

[80] Amodio P, Del Piccolo F, Pettenò E et al. Prevalence and prognostic value of quantified electroencephalogram (EEG) alterations in cirrhotic patients. J. Hepatol. 2001; 35: 37-45.

[81] Mehndiratta MM, Sood GK, Sarin SK, Gupta M: Comparative evaluation of visual, somatosensory, and auditory evoked potentials in the detection of subclinical hepatic encephalopathy in patients with nonalcoholic cirrhosis. Am J Gastroenterol 1990; 85: 799-803

[82] Saxena N, Bhatia M, Joshi YK, Garg PK, Dwivedi SN, Tandon RK. Electrophysiological and neuropsychological tests for the diagnosis of subclinical hepatic encephalopathy and prediction of overt encephalopathy. Liver. 2002; 22: 190-7. 
[83] Amodio P, Valenti P, Del Piccolo F et al. P300 latency for the diagnosis of minimal hepatic encephalopathy: evidence that spectral EEG analysis and psychometric tests are enough. Dig. Liver. Dis. 2005; 37: 861-8.

[84] Amodio P, Gatta A. Neurophysiological investigation of hepatic encephalopathy. Metab. Brain. Dis. 2005; 20: 369-79.

[85] Saxena N, Bhatia M, Joshi YK et al. Auditory P300 event-related potentials and number connection test for evaluation of subclinical hepatic encephalopathy in patients with cirrhosis of the liver: a follow-up study. J. Gastroenterol. Hepatol. 2001; 16: 322-7.

[86] Kircheis G, Wettstein M, Timmermann L et al. Critical flicker frequency for quantification of low-grade hepatic encephalopathy. Hepatology 2002; 35: 357-66.

[87] Sharma P, Sharma BC, Puri V, Sarin SK. Critical fl icker frequency: diagnostic tool for minimal hepatic encephalopathy. J Hepatol 2007;47:67-73.

[88] Bajaj JS, Hafeezullah M, Franco J et al. Inhibitory control test for the diagnosis of minimal hepatic encephalopathy. Gastroenterology 2008; 135: 1591-600.

[89] Binesh N, Huda A, Bugbee $\mathrm{M}$ et al. Adding another spectraldimension to $1 \mathrm{H}$ magnetic resonance spectroscopy of hepatic encephalopathy. J. Magn. Reson. Imaging. 2005; 21:398-405.

[90] Weissenborn K, Ehrenheim C, Hori A, Kubicka S, Manns MP.Pallidal lesions in patients with liver cirrhosis: clinical and MRI evaluation. Metab. Brain. Dis. 1995; 10: 219-31.

[91] Rose C, Butterworth RF, Zayed J et al. Manganese deposition in basal ganglia structures results from both portal-systemic shunting and liver dysfunction. Gastroenterology 1999; 117: 640-4.

[92] Yadav SK, Srivastava A, Srivastava A et al. Encephalopathy assessment in children with extra-hepatic portal vein obstruction with MR, psychometry and critical flicker frequency. J. Hepatol. 2010; 216: 683-91.

[93] Naegele T, Grodd W, Viebahn R et al. MR imaging and (1)H spectroscopy of brain metabolites in hepatic encephalopathy: time-course of renormalization after liver transplantation. Radiology 2000; 52: 348-54.

[94] Nie YQ, Zeng Z, Li YY, Sha WH, Ping L, Dai SJ. Long-term efficacy of lactulose in patients with subclinical hepatic encephalopathy. Zhonghua. Nei. Ke. Za. Zhi. 2003; 42: 261-263

[95] Weissenborn K, Ahl B, Fischer-Wasels D et al. Correlations between magnetic resonance spectroscopy alterations and cerebral ammonia and glucose metabolism in cirrhotic patients with and without hepatic encephalopathy. Gut. 2007; 56: 1736-42.

[96] Lodi R, Tonon C, Stracciari A et al. Diffusion MRI shows increased water apparent diffusion coefficient in the brains of cirrhotics. Neurology 2004; 62: 762-6.

[97] Kale RA, Gupta RK, Saraswat VA et al. Demonstration of interstitial cerebral edema with diffusion tensor MR imaging in type $C$ hepatic encephalopathy. Hepatology 2006; 43: 698-706.

[98] Caron M-J et al. Brain 2006; 129: 1789-1802

[99] Ferenci P, Lockwood A, Mullen K, Tarter R, Weissenborn K, Blei AT. Hepatic encephalopathy - definition, nomenclature, diagnosis, and quantification: fi nal report of the working party at the 11th World Congresses of Gastroenterology, Vienna, 1998. Hepatology 2002; 35: 716-721

[100] Das A, Dhiman RK, Saraswat VA, Naik SR. Prevalence and natural history of subclinical hepatic encephalopathy in cirrhosis. J Gastroenterol Hepatol 2001; 16: 531-535 


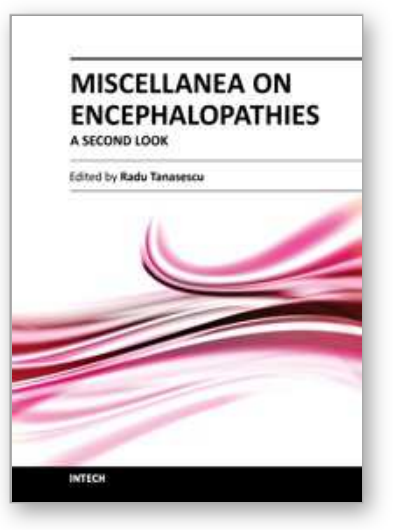

\author{
Miscellanea on Encephalopathies - A Second Look \\ Edited by Dr. Radu Tanasescu
}

ISBN 978-953-51-0558-9

Hard cover, 390 pages

Publisher InTech

Published online 25, April, 2012

Published in print edition April, 2012

The book project "Miscellanea on Encephalopathies-a second look" aims to cover some of the important aspects regarding metabolic, hypoxic, neoplasm- and drug-related encephalopathies, by transmitting valuable information filtered through the real life clinical and research experience of the authors.

\title{
How to reference
}

In order to correctly reference this scholarly work, feel free to copy and paste the following:

Daniela Benedeto-Stojanov and Dragan Stojanov (2012). Minimal Hepatic Encephalopathy (MHE), Miscellanea on Encephalopathies - A Second Look, Dr. Radu Tanasescu (Ed.), ISBN: 978-953-51-0558-9, InTech, Available from: http://www.intechopen.com/books/miscellanea-on-encephalopathies-a-second-look/minimalhepatic-encephalopathy

\section{INTECH}

open science | open minds

\section{InTech Europe}

University Campus STeP Ri

Slavka Krautzeka 83/A

51000 Rijeka, Croatia

Phone: +385 (51) 770447

Fax: +385 (51) 686166

www.intechopen.com

\section{InTech China}

Unit 405, Office Block, Hotel Equatorial Shanghai

No.65, Yan An Road (West), Shanghai, 200040, China 中国上海市延安西路65号上海国际贵都大饭店办公楼 405 单元

Phone: $+86-21-62489820$

Fax: +86-21-62489821 
(C) 2012 The Author(s). Licensee IntechOpen. This is an open access article distributed under the terms of the Creative Commons Attribution 3.0 License, which permits unrestricted use, distribution, and reproduction in any medium, provided the original work is properly cited. 